\title{
Design and Implementation of Networked ATS Based on Function Interface
}

\author{
Li Wanling ${ }^{\text {a }}$, Wang Zhensheng, Song Xiangjun and Liu Yanhong \\ Ordnance Technology research institute. Shijiazhuang Hebei, China \\ azyh8282180@sina.com
}

Keywords: function interface; networked ATS; TPS; COM

\begin{abstract}
Based on the principle of IVI, IVI-MSS and IVI-Signal, this paper has proposed a conception of function interface based on COM technology, and introduced its designing and model structure. Networked ATS was designed and implemented based on function interface and the design thinking of COM and module.
\end{abstract}

\section{Introduction}

Research and application of ATS based on network are greatly concentrated on along with the developing of auto test technology and networked technology. Establishing ATS based on network becomes a trend of development in order to adapting to the development of information technology, making full use of network agent, implementing sharing resource of expert, cooperating test and diagnosing online, and breaking through time and space. If test condition changes and test hardware is replaced, test program sets (TPS) needs exploited again, and exploitation and maintenance of ATS become more complex and difficult. So, transportability and reusability of TPSs, interchangeability of instrument become much more important. Considering those reasons, this paper puts forward networked function interface based on component to establish networked ATS.

\section{Function Interface Model}

The function interface model based on component technology was brought forward at the basis of IVI, IVI-MSS and IVI-Signal, which has defect in instrument interchangeability, TPSs transplantability and reusability, and the model was intended to solve the problem.

Design Thinking. The interface model based on COM technology treats the ability to test/stimulate signal as basic unit ignoring specific instrument. The model only considers the required function of the instrument, despite its type and manufacturer. The key to realize ATS instrument interchangeability and TPSs transplantability and reusability is the creation of abstract layer between hardware and software, which makes instruments and test programs absolutely insulated. The TPSs is totally UUT oriented, and describes test requirement and process at the basis of signals, the same basis at which bottom layer drives and calls instruments. The model is encapsulated in form of remote process outer component to produce .EXE files which can be called remotely or locally.

The Configuration of the Model. The function interface model based on COM technology is based on test signal, the test programs are UUT oriented, which means the test programs do not manipulate instruments directly, but create a function layer between test programs and actual instrument manipulation, which avoids the operation on actual instruments by test programs, negating the influence of instruments on test programs. The whole process ensures the independence of test programs and actual instruments, then the update of programs or change of instruments becomes very simple. The model comprises of four parts, which are function proxy, resource management engine, function driver model and signal channel control, the architecture is as shown in Figure 1. 


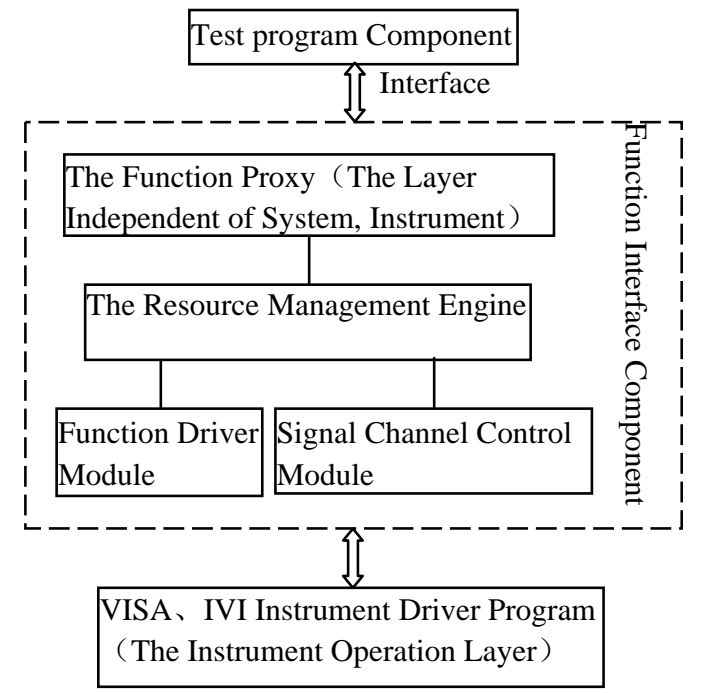

Fig.1 The Architecture of the Function Interface Based on Component

The function proxy layer knows nothing about the specific instruments, it only describes the common attributes of the functions of instruments, and these attributes are all about the process of test and result verifying. The development of TPS is totally UUT oriented, absolutely insulated from the test system; the resource management engine provides automated resource allocation and switch path calculation service, which is the key to realization of effective mapping from virtual resources to real resources; the signal oriented control of instruments and switches is completed by function driver module and signal channel control module, the former encapsulates the drivers of all instruments in the system by function, in spite of their realization, which is the basis to instrument interchangeability; the latter encapsulates the realization of switch control, and provide outer call in component form, whose interface parameters only relate to the name of UUT signal ports which need electric connections, which are thus made the basis of control to signal channel by test program, and thus realized the independence of test program between system signal channel electric connections.

Realization Principle of Instruments Interchangeability. The main thinking of function interface technology based on component is establishing function interface layer between test flow and control of actual instrument, and isolating control of instrument and test program completely. So, it can be created with these basic function interface models whether instruments are upgraded and exchanged, nor new instrument appears.

For example, a signal generator can generate five types pumping signal: direct current, sine, square wave and random waveform. According to signal pumping function, the instrumental driver can be implemented by these five function driving module. If output of $\mathrm{D} / \mathrm{A}$ can also generate these pumping signals, it has the same function driving interface, and the driver of instrument can be implemented by these five function driving module too. When the requirement of system resource is sine wave signal, signal generator and $\mathrm{D} / \mathrm{A}$ both can generate these pumping functions, and either can be chosen. It shows only function interface to test systems, and screens the difference of instruments. The isolating layer is established between instruments and test programs, which makes instruments and test programs absolutely insulated, and ensures the instrument interchangeability of TPS.

\section{Networked ATS Based on Function Interface Model}

In this paper, design and implementation of networked ATS based on function interface were modeled and studied with some antitank missile and the implication of design thinking for function interface.

Framework of System Hardware. The blue print for framework of system hardware is "test servers system + bus card instruments system +GPIB bus instruments+ linker+ adapter”. The design thinking of Hierarchy based on COM and model is adopted. Its function parts adopted standard and 
protocol used widely, then they could be used in different system, and the software could be transplanted conveniently. Its interface also agreed to the standard and protocol, and the performance of system could be advanced and enlarged by inserting new function parts. The system adopted $\mathrm{C} / \mathrm{S}$ and $\mathrm{B} / \mathrm{S}$ framework and Intranet, and was composed of test servers, clients, Web servers and data servers, and shown as figure 2.

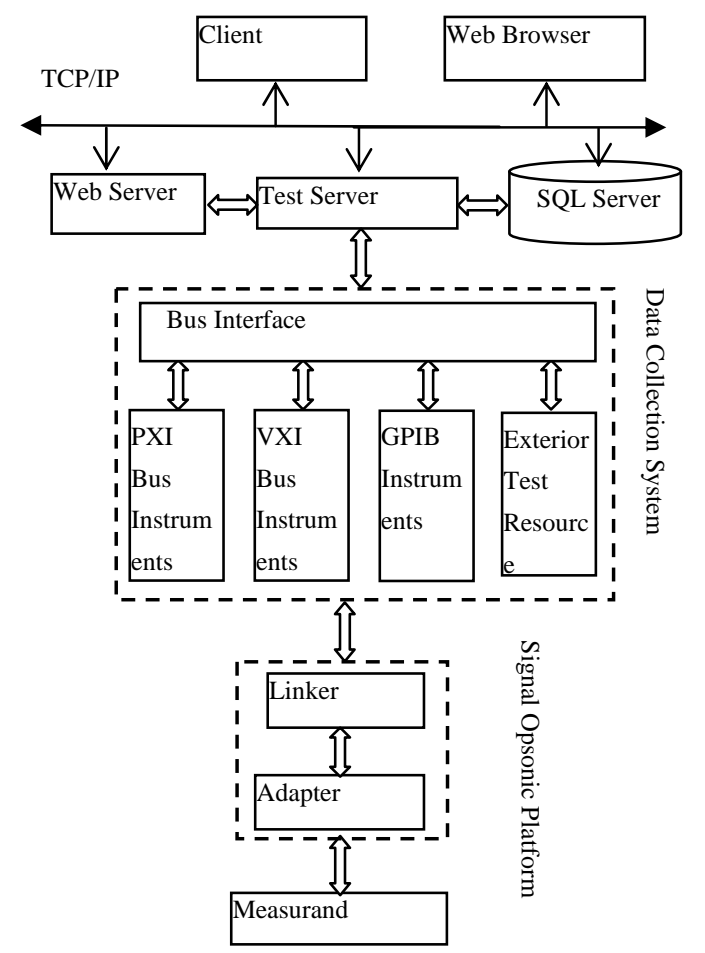

Figure 2 The Hardware Architecture of System

Framework of System Software. The platform of system software adopted architecture of layer based on COM, meanwhile used function interface. The system software was composed of COM of controlling data collection, COM of analyzing and processing data, COM of database, COM of diagnosing fault and COM of function interface, and shown as figure 3.

Implementation of System. Function interface component, FUNCTION_INTE_SERVER, is exploited in this paper to test a certain unit for the test of some missile. As length of the paper is limited, the code was not shown.

The Function interface was called in a certain unit for the test of some missile, and part of its code as follows.

IFUNCTION_INTE_SERVER *pICdata=NULL;

void _fastcall TForm1::FormCreate(TObject *Sender)

\{ OleInitialize(NULL);

HRESULT

hr=CoCreateInstance(CLSID_FUNCTION_INTE_SERVER

_,NULL,CLSCTX_SERVER,IID_IFUNCTION_INTE_SERVER,(VOID**)\&run);

if (SUCCEEDED(hr))

MessageBox(Handle,"Running","OK",MB_OK);

pICdata ->Run(5);

pICdata r->ShowKMQ;

pICdata ->Release; $\}$ 


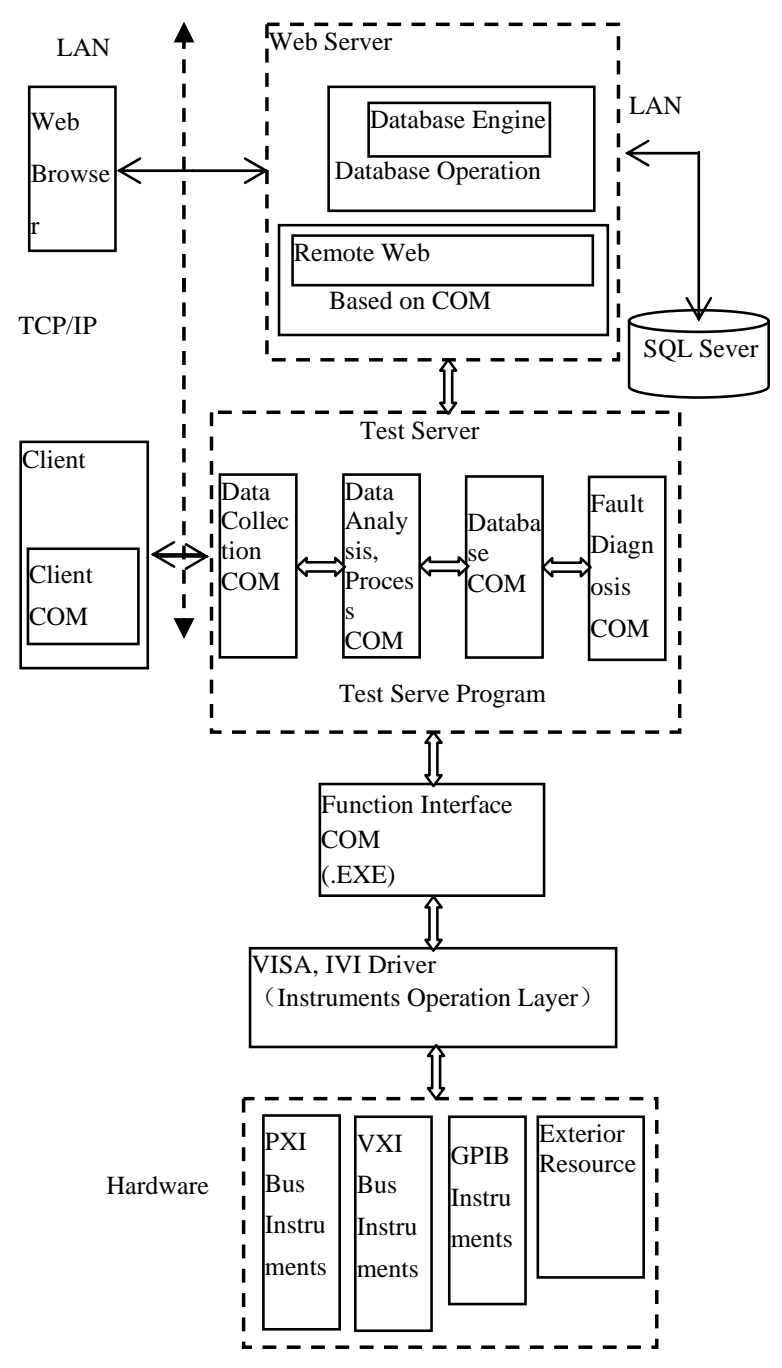

Fig.3 The Software Architecture of System

\section{Summary}

Based on the principle of IVI, IVI-MSS and IVI-Signal, making use of component technology, this thesis proposed a software design thinking of function interface based on component technology, this paper has proposed a conception of function interface based on COM technology, and studied on its model structure and the implementation of instrument interchangeability. Networked test was implemented based on function interface and the design thinking of COM and module, and had certain meaning of guidance for exploiting and designing ATS.

\section{References}

[1] Luo Jin. Research of general ATS Key Technology [D]. Mechanism Engineering College doctoral paper. 2006.

[2] Li Wanling. et Research on Function Interface Based on Component Technology. $8^{\text {th }}$ ICEMI. 2007.8:1-345-1-348.

[3] Zhao Huibing Virtual Instrument Specification and System Integration.tsinghua.edu, 2003.8.

[4] An Youlin .et.Design and implementation of Networked test system based on LabWindows/CVI and C++Builder. computer engineering and design[J].2005,11(26):3042-3044.

[5] Liu Jinning,. et Software Design of Virtual Instruments Based on Function Interface. Electrical Measurement \& Instrument. 2005,42(7): 54-57. 